\title{
Stability Analysis of Bubonic Plague Model with the Causing Pathogen Yersinia pestis in the Environment
}

\author{
Rigobert Charles Ngeleja $^{1^{*}}$, Livingstone Luboobi ${ }^{2}$, Yaw Nkansah-Gyekye ${ }^{1}$ \\ ${ }^{1}$ School of Computational and Communication Science and Engineering, Nelson Mandela African Institution of \\ Science and Technology, Arusha, Tanzania \\ ${ }^{2}$ Department of Mathematics, Makerere University, Kampala, Uganda \\ Email: *rngeleja@yahoo.com, yaw.nkansah-gyekye@nm-aist.ac.tz, luboobi@cns.mak.ac.ug
}

Received 23 June 2016; accepted 13 September 2016; published 16 September 2016

Copyright @ 2016 by authors and Scientific Research Publishing Inc.

This work is licensed under the Creative Commons Attribution International License (CC BY).

http://creativecommons.org/licenses/by/4.0/

(c) (i) Open Access

\section{Abstract}

Bubonic plague is a serious bacterial disease, mainly transmitted to human beings and rodents through flea bite. However, the disease may also be transmitted upon the interaction with the infected materials or surfaces in the environment. In this study, a deterministic model for bubonic plague disease with Yersinia pestis in the environment is developed and analyzed. Conditions for existence and stability of the equilibrium points are established. Using Jacobian method disease free equilibrium (DFE) point, $E^{0}$ was proved to be locally asymptotically stable. The Metzler matrix method was used to prove that the DFE was globally asymptotically stable when $R_{0}<1$. By applying Lyapunov stability theory and La Salles invariant principle, we prove that the endemic equilibrium point of system is globally asymptotically stable when $R_{0}>1$. Numerical simulations are done to verify the analytical predictions. The results show that bubonic plague can effectively be controlled or even be eradicated if efforts are made to ensure that there are effective and timely control strategies.

\section{Keywords}

Disease Free Equilibrium, Endemic Equilibrium Stability Analysis, Bubonic Plague, Pathogens in the Environment

\section{Introduction}

Bubonic plague is the bacterial infection caused by Yersinia pestis when the bacteria infect lymphatic system [1].

${ }^{*}$ Corresponding author.

How to cite this paper: Ngeleja, R.C., Luboobi, L. and Nkansah-Gyekye, Y. (2016) Stability Analysis of Bubonic Plague Model with the Causing Pathogen Yersinia pestis in the Environment. Advances in Infectious Diseases, 6, 120-137. 
It is characterised by geographical foci and extraordinarily adaptation capability which gives it ability to reemerge even after decades of silence. Thus even though the disease is historic it still infect and kill thousands of people around the world [2].

The disease mainly affects wild rodents, it can also be transmitted to human and other domestic animals through flea bites. Bubonic plague causes fever and very throbbing swelling of the lymph glands also called buboes, which is the reason why the disease is called bubonic plague.

When the flea is infested with pathogens causing bubonic plague the bacteria multiply in the proventriculus (foregut) of the flea [3]. The bacteria have the tendency of blocking the flea's bloodsucking apparatus which consequently lead to inability of flea to pump blood into the midgut for digestion. This makes the flea to become ravenous as a result flea bites the host repetitively while vomiting the bacteria causing disease into the host. When a host dies, fleas move off the body to seek another live warm-blooded host [4].

Although it is not yet clearly known how, but Yersinia pestis may survive in the soil and remain viable and fully virulent for 40 weeks in soil and can cause the infection upon the adequate interaction with the susceptible individual. This is believed to be the reason for possible mechanism of interepizootic persistence, epizootic spread, and as a factor defining plague foci [5].

In this paper, we discuss the stability analysis of the bubonic plague epidemic model in human, rodent and flea population. The model includes the transmission from the environment to the susceptible human or rodent. We also discuss the disease-free equilibrium point, endemic equilibrium point of the model and analyze the local and global stability of these steady states. We finally use the numerical simulation to support our analytical results.

\section{Model Formulation}

This paper presents the stability analysis of the bubonic plague epidemic model developed by [6]. The model includes four interacting population which are: human population, Flea population, Rodent population and pathogens in the environment is developed. We use $S_{H}, E_{H}, I_{H}$ and $R_{H}$ to represent Susceptible human beings, Exposed human beings, Infected human beings and Recovered human beings respectively; $S_{R}, E_{R}$ and $I_{R}$ for Susceptible rodents, Exposed rodents and Infected rodents respectively. The Susceptible and the Infectious flea are denoted by $S_{F}$ and $I_{F}$ respectively. The pathogens in the environment are denoted by $A$. The total population for human being, rodent and flea population is by

$$
\begin{aligned}
& N_{1}=S_{H}+E_{H}+I_{H}+R_{H} \\
& N_{2}=S_{F}+I_{F} \\
& N_{3}=S_{R}+E_{R}+I_{R}
\end{aligned}
$$

The parameters used in the model are described in Table 1.

\section{Model Equations for Bubonic Plague}

Since we allow the population in and out of the compartments, the rate at which new infections occur in a population will depend on the fraction of the population that is infected (disease prevalence). The infection rate in human depends on the probability that a contact between infectious flea and susceptible human and between infectious environment and susceptible human leads to infection. For the rodent the infection depends on the probability that a contact between infectious flea and susceptible rodent and between infectious environment and susceptible rodent leads to infection. For the flea the infection depends on the probability that a contact between infectious human and susceptible flea and between infectious rodent and susceptible flea leads to infection. Therefore the infection rates of susceptible humans, rodent population and flea population are as given in (2a), (2b) and (2c) respectively.

$$
\begin{aligned}
& \Gamma_{f h} \frac{I_{F}}{N_{2}}+\omega_{1} A \\
& \Gamma_{f r} \frac{I_{F}}{N_{2}}+\omega_{2} A
\end{aligned}
$$


Table 1. Parameters and their description.

\begin{tabular}{|c|c|}
\hline Parameters & Description \\
\hline$\Gamma_{r f}$ & Adequate contact rate: infected rodent to flea \\
\hline$\Gamma_{f h}$ & Adequate contact rate: infected flea to human \\
\hline$\Gamma_{f r}$ & Adequate contact rate: infected flea to rodent \\
\hline$\alpha_{1}$ & Progression rate of susceptible human to exposed \\
\hline$\gamma_{1}$ & Progression rate of susceptible rodent to exposed \\
\hline$\Gamma_{h f}$ & Adequate contact rate: infected human to flea \\
\hline$\lambda_{4}$ & Recruitment rate of pathogens \\
\hline$\alpha_{2}$ & Progression rate of exposed human to infected \\
\hline$\gamma_{2}$ & Progression rate of exposed rodent to infected \\
\hline$\alpha_{3}$ & Human recovery rate \\
\hline$\varpi$ & Progression rate of recovered human to susceptible \\
\hline$\mu_{1}$ & Natural death rate for Human \\
\hline$\delta_{1}$ & Disease induced death rate for Human \\
\hline$\delta_{3}$ & Disease induced death rate for rodent \\
\hline$\mu_{3}$ & Natural death rate for rodent \\
\hline$\omega_{1}$ & Adequate contact rate: Pathogens to human \\
\hline$\omega_{2}$ & Adequate contact rate: Pathogens to rodent \\
\hline$\mu_{4}$ & Natural death rate for Pathogens \\
\hline$\mu_{2}$ & Natural death rate for flea \\
\hline$\delta_{2}$ & Disease induced death rate for flea \\
\hline$\psi_{1}$ & Immigration rate of human \\
\hline$\psi_{2 s}$ & Immigration rate of Susceptible flea \\
\hline$\psi_{2 i}$ & Immigration rate of Infected flea \\
\hline$\psi_{3}$ & Immigration rate of rodent \\
\hline$\pi_{1}$ & The proportion of human migrants that are Susceptible \\
\hline$\pi_{2}$ & The proportion of human migrants that are Exposed \\
\hline$\pi_{3}$ & The proportion of human migrants that are Recovered \\
\hline$\kappa_{1}$ & The proportion of rodent migrants that are Susceptible \\
\hline$\kappa_{2}$ & The proportion of rodent migrants that are Exposed \\
\hline$\kappa_{3}$ & The proportion of rodent migrants that are Infected \\
\hline$\beta$ & The rate at which fleas become infected \\
\hline
\end{tabular}




$$
\rho \Gamma_{h f} \frac{I_{H}}{N_{1}}+(1-\rho) \Gamma_{r f} \frac{I_{r}}{N_{3}}
$$

Pathogens in the environment are recruited at a constant rate $\lambda_{4}$ and they are removed through natural death $\mu_{4}$ or removed when they contact with susceptible human and rodent at the rates $\omega_{1}$ and $\omega_{2}$ respectively.

Using the definition of variables and parameters stated in Table 1, we drive model for the dynamics of bubonic plague disease in human, rodent, flea and pathogens in the environment as given in (3), (4), (5) and (6) respectively.

Human

$$
\begin{aligned}
& \frac{\mathrm{d} S_{H}}{\mathrm{~d} t}=\pi_{1} \psi_{1}+\varpi R_{H}-\alpha_{1}\left(\Gamma_{f h} \frac{I_{F}}{N_{2}}+\omega_{1} A\right) S_{H}-\mu_{1} S_{H}, \\
& \frac{\mathrm{d} E_{H}}{\mathrm{~d} t}=\pi_{2} \psi_{1}+\alpha_{1}\left(\Gamma_{f h} \frac{I_{F}}{N_{2}}+\omega_{1} A\right) S_{H}-\alpha_{2} E_{H}-\mu_{1} E_{H}, \\
& \frac{\mathrm{d} I_{H}}{\mathrm{~d} t}=\alpha_{2} E_{H}-\alpha_{3} I_{H}-\left(\mu_{1}+\delta_{1}\right) I_{H}, \\
& \frac{\mathrm{d} R_{H}}{\mathrm{~d} t}=\pi_{3} \psi_{1}+\alpha_{3} I_{H}-\varpi R_{H}-\mu_{1} R_{H} .
\end{aligned}
$$

Rodent

$$
\begin{aligned}
& \frac{\mathrm{d} S_{R}}{\mathrm{~d} t}=\kappa_{1} \psi_{3}-\gamma_{1}\left(\Gamma_{f r} \frac{I_{F}}{N_{2}}+\omega_{2} A\right) S_{R}-\mu_{3} S_{R} \\
& \frac{\mathrm{d} E_{R}}{\mathrm{~d} t}=\kappa_{2} \psi_{3}+\gamma_{1}\left(\Gamma_{f r} \frac{I_{F}}{N_{2}}+\omega_{2} A\right) S_{R}-\gamma_{2} E_{R}-\mu_{3} E_{R} \\
& \frac{\mathrm{d} I_{R}}{\mathrm{~d} t}=\kappa_{3} \psi_{3}+\gamma_{2} E_{R}-\left(\mu_{3}+\delta_{3}\right) I_{R}
\end{aligned}
$$

Flea

$$
\begin{aligned}
& \frac{\mathrm{d} S_{F}}{\mathrm{~d} t}=\psi_{2 s}-\beta\left(\rho \Gamma_{h f} \frac{I_{H}}{N_{1}}+(1-\rho) \Gamma_{r f} \frac{I_{R}}{N_{3}}\right) S_{F}-\mu_{2} S_{F} \\
& \frac{\mathrm{d} I_{F}}{\mathrm{~d} t}=\psi_{2 i}+\beta\left(\rho \Gamma_{h f} \frac{I_{H}}{N_{1}}+(1-\rho) \Gamma_{r f} \frac{I_{R}}{N_{3}}\right) S_{F}-\left(\mu_{2}+\delta_{2}\right) I_{F}
\end{aligned}
$$

where $\psi_{2 s}<\psi_{2 i}$

Pathogens

$$
\frac{\mathrm{d} A}{\mathrm{~d} t}=\lambda_{4}-\omega_{1} A S_{H}-\omega_{2} A S_{R}-\mu_{4} A
$$

\section{Steady State and Local Stability of the Critical Points}

In this section we consider existence of equilibrium states and stability of the equilibrium states of the system (3)-(6).

\subsection{Disease Free Equilibrium}

The model has disease free equilibrium which is obtained by setting $I_{H}=E_{H}=R_{H}=0, \quad I_{R}=E_{R}=0, \quad I_{F}=0$ and $A=0$ and the derivatives equal to zero into the system (3)-(6). 
Then we have the disease free-equilibrium point given as $E_{H}^{0}=\left(\frac{\pi_{1} \psi_{1}}{\mu_{1}}, 0,0,0\right), E_{R}^{0}=\left(\frac{\kappa_{1} \psi_{3}}{\mu_{3}}, 0,0\right)$, $E_{F}^{0}=\left(\frac{\psi_{2 s}}{\mu_{2}}, 0\right)$ and $E_{A}^{0}=0$ for Human, Rodent, Flea and Pathogen respectively.

Then the disease free equilibrium of the entire system is

$$
E^{0}\left(S_{H}^{0}, E_{H}^{0}, I_{H}^{0}, R_{H}^{0}, S_{R}^{0}, E_{R}^{0}, I_{R}^{0}, S_{F}^{0}, I_{F}^{0}, A^{0}\right)=\left(\frac{\pi_{1} \psi_{1}}{\mu_{1}}, 0,0,0, \frac{\kappa_{1} \psi_{3}}{\mu_{3}}, 0,0, \frac{\psi_{2 s}}{\mu_{2}}, 0,0\right)
$$

\subsection{Local Stability of the Disease-Free Equilibrium Point}

In this section we consider the local stability analysis of the disease free equilibrium point of the bubonic plague disease system (3)-(6). We analyze the local stability of the disease free equilibrium point using the Jacobian method in which all equations in system (3)-(6) are considered and analyzed at the disease free equilibrium $E^{0}$. In this method we compute and examine the eigenvalues of Jacobian matrix of the system (3)-(6) to prove that the DFE is locally and asymptotically stable. We are required to show that all real parts of the eigenvalues at $E^{0}$ are negative. Now in order to attest that the eigenvalues are negative we need to prove the general condition that the determinant and the trace of the Jacobian matrix are positive and negative respectively [7].

Now the Jacobian matrix of the system (3)-(6) at $E^{0}$ is given by:

$$
\boldsymbol{J}\left(E^{0}\right)=\left(\begin{array}{cccccccccc}
-\mu_{1} & 0 & 0 & \varpi & 0 & 0 & 0 & 0 & -k_{3} & \frac{-\alpha_{1} \pi_{1} \psi_{1} \omega_{1}}{\mu_{1}} \\
0 & -k_{7} & 0 & 0 & 0 & 0 & 0 & 0 & k_{3} & \frac{\alpha_{1} \pi_{1} \psi_{1} \omega_{1}}{\mu_{1}} \\
0 & \alpha_{2} & -k_{6} & 0 & 0 & 0 & 0 & 0 & 0 & 0 \\
0 & 0 & \alpha_{3} & -k_{9} & 0 & 0 & 0 & 0 & 0 & 0 \\
0 & 0 & 0 & 0 & -\mu_{3} & 0 & 0 & 0 & -k_{4} & \frac{-\gamma_{1} \kappa_{1} \psi_{3} \omega_{2}}{\mu_{3}} \\
& & & & & & & & & \frac{\gamma_{1} \kappa_{1} \psi_{3} \omega_{2}}{\mu_{3}} \\
0 & 0 & 0 & 0 & 0 & -k_{10} & 0 & 0 & k_{4} \\
0 & 0 & 0 & 0 & 0 & \gamma_{2} & -k_{11} & 0 & 0 & 0 \\
0 & 0 & -k_{1} & 0 & 0 & 0 & -k_{2} & -\mu_{2} & 0 & 0 \\
0 & 0 & k_{1} & 0 & 0 & 0 & k_{2} & 0 & -k_{8} & 0 \\
0 & 0 & 0 & 0 & 0 & 0 & 0 & 0 & 0 & -k_{5}
\end{array}\right)
$$

where

$$
\begin{array}{lll}
k_{1}=\frac{\beta \rho \Gamma_{h f} \psi_{2 s} \mu_{1}}{\pi_{1} \psi_{1} \mu_{2}} & k_{2}=\frac{\beta(1-\rho) \Gamma_{r f} \psi_{2 s} \mu_{3}}{\kappa_{1} \psi_{3} \mu_{2}} & k_{3}=\frac{\alpha_{1} \pi_{1} \psi_{1} \mu_{2} \Gamma_{f h}}{\mu_{1} \psi_{2 s}} \\
k_{4}=\frac{\gamma_{1} \kappa_{1} \psi_{3} \mu_{2} \Gamma_{f r}}{\mu_{3} \psi_{2 s}} & k_{5}=\omega_{1}+\omega_{2}+\mu_{4} & k_{6}=\alpha_{3}+\mu_{1}+\delta_{1} \\
k_{7}=\alpha_{2}+\mu_{1} & k_{8}=\mu_{2}+\delta_{2} & k_{9}=\varpi+\mu_{1} \\
k_{10}=\gamma_{2}+\mu_{3} & k_{11}=\mu_{3}+\delta_{3} &
\end{array}
$$

We now use Trace and determinant method to check the stability of the disease free equilibrium point $E^{0}$ in which we need to prove that the trace and the determinant of matrix (7) are negative and positive respectively

Then using mathematica software we prove that trace of the matrix (7) given by

$$
\text { Trace }=-\mu_{1}-\left(\alpha_{2}+\mu_{1}\right)-k_{6}-\left(\varpi+\mu_{1}\right)-\mu_{3}-\left(\gamma_{2}+\mu_{3}\right)-\left(\mu_{3}+\delta_{3}\right)-\mu_{2}-\left(\mu_{2}+\delta_{2}\right)-k_{5}
$$


where

$$
k_{5}=\omega_{1}+\omega_{2}+\mu_{4}, \quad k_{6}=\alpha_{3}+\mu_{1}+\delta_{1}
$$

It is clear that the trace of the matrix (7) in negative. Then using the same software (mathematica) we are able to prove that the determinant of the matrix (7) is positive provided:

$$
\sqrt{\frac{\beta}{\left(\mu_{2}+\delta_{2}\right)}\left(\frac{\gamma_{2} \gamma_{1} \Gamma_{r f} \Gamma_{f r}(1-\rho)}{\left(\mu_{3}+\gamma_{2}\right)\left(\mu_{3}+\delta_{3}\right)}+\frac{\rho \alpha_{2} \alpha_{1} \Gamma_{h f} \Gamma_{f h}}{\left(\alpha_{2}+\mu_{1}\right)\left(\alpha_{3}+\mu_{1}+\delta_{1}\right)}\right)}<1
$$

where

$$
\sqrt{\frac{\beta}{\left(\mu_{2}+\delta_{2}\right)}\left(\frac{\gamma_{2} \gamma_{1} \Gamma_{r f} \Gamma_{f r}(1-\rho)}{\left(\mu_{3}+\gamma_{2}\right)\left(\mu_{3}+\delta_{3}\right)}+\frac{\rho \alpha_{2} \alpha_{1} \Gamma_{h f} \Gamma_{f h}}{\left(\alpha_{2}+\mu_{1}\right)\left(\alpha_{3}+\mu_{1}+\delta_{1}\right)}\right)}
$$

is the basic reproduction number, $R_{0}$.

$R_{0}$ measures the average number of secondary infection produced when a typical infectious individual enters an entirely susceptible population. In our case, due to presence of multiple transmission cycle the basic reproductive number do not give the number of cases infected by a single individual but rather the geometric mean of the number of infections per generation [8].

Referring to (8), the geometric mean of the number of infections per generation depends on: rodent's infective period $\frac{1}{\mu_{3}+\delta_{3}}$, the probability that flea gets the disease from the rodent or human which are $(1-\rho) \Gamma_{r f}$ or $\rho \Gamma_{h f}$ respectively The human infective period $\frac{1}{\mu_{1}+\delta_{1}+\alpha_{3}}$, probability that human survive the infected class $\frac{\alpha_{2}}{\mu_{1}+\alpha_{2}}$, the rate at which fleas gets infected $\beta$, flea's infective period $\frac{1}{\mu_{2}+\delta_{2}}$, probability that rodent survive the infected class $\frac{\gamma_{2}}{\mu_{3}+\gamma_{2}}$, the adequate contact rate flea to human $\Gamma_{f h}$, the adequate contact rate flea to rodent $\Gamma_{f r}$ and the rate at which human and rodent become exposed to the the disease which are $\alpha_{1}$ and $\gamma_{1}$ respectively.

Thus disease free equilibrium point $E^{0}$ is therefore locally asymptotically stable and leads to the following theorem:

Theorem 1. The Disease Free Equilibrium $E^{0}$ of bubonic plague is locally asymptotically stable if $R_{0}<1$ and unstable if $R_{0}>1$.

\subsection{Global Stability of the Disease-Free Equilibrium Point}

In this section we analyze the global stability of the disease free equilibrium point using Metzler matrix method as stated by [9]. To do this we first sub-divide the general system (3)-(6) of bubonic plague disease into transmitting and non-transmitting component.

Now let $\boldsymbol{Y}_{n}$ be the vector for non-transmitting compartment, $\boldsymbol{Y}_{i}$ be the vector for transmitting compartment and $\boldsymbol{Y}_{E_{0}, n}$ be the vector of disease free point. Then

$$
\left\{\begin{array}{l}
\frac{\mathrm{d} \boldsymbol{Y}_{n}}{\mathrm{~d} t}=\boldsymbol{A}_{1}\left(\boldsymbol{Y}_{n}-\boldsymbol{Y}_{E_{0}, n}\right)+\boldsymbol{A}_{2} \boldsymbol{Y}_{i} \\
\frac{\mathrm{d} \boldsymbol{Y}_{i}}{\mathrm{~d} t}=\boldsymbol{A}_{3} \boldsymbol{Y}_{i}
\end{array}\right.
$$

We then have

$$
\boldsymbol{Y}_{n}=\left(S_{H}, R_{H}, S_{R}, S_{F}\right)^{\mathrm{T}} \quad \boldsymbol{Y}_{i}=\left(E_{H}, I_{H}, E_{R}, I_{R}, I_{F}, A\right) \quad \boldsymbol{Y}_{E_{0}, n}=\left(\frac{\pi_{1} \psi_{1}}{\mu_{1}}, 0, \frac{\kappa_{1} \psi_{3}}{\mu_{3}}, \frac{\psi_{2 s}}{\mu_{2}}\right)
$$




$$
\boldsymbol{Y}_{n}-\boldsymbol{Y}_{E_{0}, n}=\left(\begin{array}{c}
S_{H}-\frac{\pi_{1} \psi_{1}}{\mu_{1}} \\
R_{H} \\
S_{R}-\frac{\kappa_{1} \psi_{3}}{\mu_{3}} \\
S_{F}-\frac{\psi_{2 s}}{\mu_{2}}
\end{array}\right)
$$

Now to prove the global stability of the DFE we need to show that Matrix $\boldsymbol{A}_{1}$ has real negative eigenvalues and $\boldsymbol{A}_{3}$ is a Metzler matrix in which all off diagonal element must be non-negative. Referring to (9) we write the general model as given below

$$
\left(\begin{array}{c}
\pi_{1} \psi_{1}+\varpi R_{H}-\alpha_{1} k S_{H}-\mu_{1} S_{H}, \\
\pi_{3} \psi_{1}+\alpha_{3} I_{H}-\varpi R_{H}-\mu_{1} R_{H} \cdot \\
\kappa_{1} \psi_{3}-\gamma_{1} M S_{R}-\mu_{3} S_{R} \\
\psi_{2 s}-\beta Y S_{F}-\mu_{2} S_{F}
\end{array}\right)=A_{1}\left(\begin{array}{c}
S_{H}-\frac{\pi_{1} \psi_{1}}{\mu_{1}} \\
R_{H} \\
S_{R}-\frac{\kappa_{1} \psi_{3}}{\mu_{3}} \\
S_{F}-\frac{\psi_{2 s}}{\mu_{2}}
\end{array}\right)+A_{2}\left(\begin{array}{c}
E_{H} \\
I_{H} \\
E_{R} \\
I_{R} \\
I_{F} \\
A
\end{array}\right)
$$

and

$$
\left(\begin{array}{c}
\pi_{2} \psi_{1}+\alpha_{1} k S_{H}-\alpha_{2} E_{H}-\mu_{1} E_{H}, \\
\alpha_{2} E_{H}-\alpha_{3} I_{H}-\left(\mu_{1}+\delta_{1}\right) I_{H}, \\
\kappa_{2} \psi_{3}+\gamma_{1} M S_{R}-\gamma_{2} E_{R}-\mu_{3} E_{R} \\
\kappa_{3} \psi_{3}+\gamma_{2} E_{R}-\left(\mu_{3}+\delta_{3}\right) I_{R} \\
\psi_{2 i}+\beta Y S_{F}-\left(\mu_{2}+\delta_{2}\right) I_{F} \\
\lambda_{4}-\omega_{1} A S_{H}-\omega_{2} A S_{R}-\mu_{4} A
\end{array}\right)=A_{3}\left(\begin{array}{c}
E_{H} \\
I_{H} \\
E_{R} \\
I_{R} \\
I_{F} \\
A
\end{array}\right)
$$

For

$$
k=\left(\Gamma_{f h} \frac{I_{F}}{N_{2}}+\omega_{1} A\right) \quad M=\left(\Gamma_{f r} \frac{I_{F}}{N_{2}}+\omega_{2} A\right) \quad Y=\left(\rho \Gamma_{h f} \frac{I_{H}}{N_{1}}+(1-\rho) \Gamma_{r f} \frac{I_{R}}{N_{3}}\right)
$$

Now using the transmitting and non-transmitting element on the general system we will have the matrices below:

$$
\begin{aligned}
& \boldsymbol{A}_{1}=\left(\begin{array}{cccc}
-\mu_{1} & \varpi & 0 & 0 \\
0 & -\left(\varpi+\mu_{1}\right) & 0 & 0 \\
0 & 0 & -\mu_{3} & 0 \\
0 & 0 & 0 & -\mu_{2}
\end{array}\right) \\
& \boldsymbol{A}_{2}=\left(\begin{array}{cccccc}
0 & 0 & 0 & 0 & \frac{-\alpha_{1} \pi_{1} \psi_{1} \mu_{2} \Gamma_{f h}}{\mu_{1} \psi_{2 s}} & \frac{-\alpha_{1} \pi_{1} \psi_{1} \omega_{1}}{\mu_{1}} \\
0 & \alpha_{3} & 0 & 0 & 0 & 0 \\
0 & 0 & 0 & 0 & \frac{-\gamma_{1} \kappa_{1} \psi_{3} \mu_{2} \Gamma_{f r}}{\mu_{3} \psi_{2 s}} & \frac{-\gamma_{1} \kappa_{1} \psi_{3} \omega_{2}}{\mu_{3}} \\
0 & \frac{-\beta \psi_{2 s} \mu_{1} \rho \Gamma_{h f}}{\mu_{2} \pi_{1} \psi_{1}} & 0 & \frac{-\beta \psi_{2 s} \mu_{3}(1-\rho) \Gamma_{r f}}{\mu_{2} \kappa_{1} \psi_{3}} & 0 & 0
\end{array}\right)
\end{aligned}
$$




$$
A_{3}=\left(\begin{array}{cccccc}
-\left(\alpha_{2}+\mu_{1}\right) & 0 & 0 & 0 & \frac{\alpha_{1} \pi_{1} \psi_{1} \mu_{2} \Gamma_{f h}}{\mu_{1} \psi_{2 s}} & \frac{\alpha_{1} \pi_{1} \psi_{1} \omega_{1}}{\mu_{1}} \\
\alpha_{2} & -\zeta_{1} & 0 & 0 & 0 & 0 \\
0 & 0 & -\left(\gamma_{2}+\mu_{3}\right) & 0 & \frac{\gamma_{1} \kappa_{1} \psi_{3} \mu_{2} \Gamma_{f r}}{\mu_{3} \psi_{2 s}} & \frac{\gamma_{1} \kappa_{1} \psi_{3} \omega_{2}}{\mu_{3}} \\
0 & 0 & \gamma_{2} & -\left(\mu_{3}+\delta_{3}\right) & 0 & 0 \\
0 & \zeta_{3} & 0 & \frac{\beta(1-\rho) \Gamma_{r f} \psi_{2 s} \mu_{3}}{\kappa_{1} \psi_{3} \mu_{2}} & -\left(\mu_{2}+\delta_{2}\right) & 0 \\
0 & 0 & 0 & 0 & 0 & -\zeta_{2}
\end{array}\right)
$$

where $\zeta_{1}=\left(\alpha_{3}+\mu_{1}+\delta_{1}\right), \quad \zeta_{2}=\left(\omega_{1} S_{H}+\omega_{2} S_{R}+\mu_{4}\right)$ and $\zeta_{3}=\frac{\beta \rho \Gamma_{h f} \psi_{2 s} \mu_{1}}{\pi_{1} \psi_{1} \mu_{2}}$.

Now when we consider matrix $\boldsymbol{A}_{1}$, the computation shows that the eigenvalues are real and negative, which now confirms that the system

$$
\frac{\mathrm{d} \boldsymbol{Y}_{n}}{\mathrm{~d} t}=\boldsymbol{A}_{1}\left(\boldsymbol{Y}_{n}-\boldsymbol{Y}_{E_{0}, n}\right)+\boldsymbol{A}_{2} \boldsymbol{Y}_{i}
$$

is globally and asymptotically stable at $\boldsymbol{Y}_{E_{0}}$. And for matrix $\boldsymbol{A}_{3}$ we find that all its off-diagonal elements are non-negative and thus $\boldsymbol{A}_{3}$ is a Metzler stable matrix.Therefore Disease Free Equilibrium point for the general bubonic plague system is globally asymptotically stable as a result we have the following theorem:

Theorem 2. The disease-free equilibrium point is globally asymptotically stable in $E_{0}$ if $R_{0}<1$ and unstable if $R_{0}>1$.

\subsection{Existence of Endemic Equilibrium}

Here we consider the situation in which the disease persist in a population. We investigate conditions for existence of the endemic equilibrium point of the system (3)-(6). The endemic equilibrium point $E^{*}\left(S_{H}^{*}, E_{H}^{*}, I_{H}^{*}, R_{H}^{*}, S_{R}^{*}, E_{R}^{*}, I_{R}^{*}, S_{F}^{*}, I_{F}^{*}, A^{*}\right)$ is obtained by solving the equations obtained by setting the derivatives of (3)-(6) equal to zero as in (13)-(16) which exist for $R_{0}>1$.

Human

$$
\begin{aligned}
& \pi_{1} \psi_{1}+\varpi R_{H}-\alpha_{1}\left(\Gamma_{f h} \frac{I_{F}}{N_{2}}+\omega_{1} A\right) S_{H}-\mu_{1} S_{H}=0 \\
& \pi_{2} \psi_{1}+\alpha_{1}\left(\Gamma_{f h} \frac{I_{F}}{N_{2}}+\omega_{1} A\right) S_{H}-\alpha_{2} E_{H}-\mu_{1} E_{H}=0 \\
& \alpha_{2} E_{H}-\alpha_{3} I_{H}-\left(\mu_{1}+\delta_{1}\right) I_{H}=0 \\
& \pi_{3} \psi_{1}+\alpha_{3} I_{H}-\varpi R_{H}-\mu_{1} R_{H}=0
\end{aligned}
$$

Rodent

$$
\begin{aligned}
& \kappa_{1} \psi_{3}-\gamma_{1}\left(\Gamma_{\text {fr }} \frac{I_{F}}{N_{2}}+\omega_{2} A\right) S_{R}-\mu_{3} S_{R}=0 \\
& \kappa_{2} \psi_{3}+\gamma_{1}\left(\Gamma_{f r} \frac{I_{F}}{N_{2}}+\omega_{2} A\right) S_{R}-\gamma_{2} E_{R}-\mu_{3} E_{R}=0 \\
& \kappa_{3} \psi_{3}+\gamma_{2} E_{R}-\left(\mu_{3}+\delta_{3}\right) I_{R}=0
\end{aligned}
$$

Flea 


$$
\begin{aligned}
& \psi_{2 s}-\beta\left(\rho \Gamma_{h f} \frac{I_{H}}{N_{1}}+(1-\rho) \Gamma_{r f} \frac{I_{R}}{N_{3}}\right) S_{F}-\mu_{2} S_{F}=0 \\
& \psi_{2 i}+\beta\left(\rho \Gamma_{h f} \frac{I_{H}}{N_{1}}+(1-\rho) \Gamma_{r f} \frac{I_{R}}{N_{3}}\right) S_{F}-\left(\mu_{2}+\delta_{2}\right) I_{F}=0
\end{aligned}
$$

where $\psi_{2 s}<\psi_{2 i}$

Pathogens

$$
\lambda_{4}-\omega_{1} A S_{H}-\omega_{2} A S_{R}-\mu_{4} A=0
$$

Since it is difficult to obtain explicitly the endemic equilibrium points of the model we will prove its existence using the study by [10] [11]. For the endemic equilibrium to exist it must satisfy the condition $E_{H} \neq 0$ or $I_{H} \neq 0$ or $E_{R} \neq 0$ or $I_{R} \neq 0$ or $I_{F} \neq 0$ or $A \neq 0$ that is $S_{H}>0$ or $E_{H}>0$ or $I_{H}>0$ or $S_{R}>0$ or $I_{R}>0$ or $E_{R}>0$ or $S_{F}>0$ or $I_{F}>0$ or $A>0$ must be satisfied. Now adding system (13)-(16) we have

$$
\begin{aligned}
& \psi_{1}+\psi_{2 s}+\psi_{2 i}+\psi_{3}+\lambda_{4}-\mu_{1}\left(S_{H}+E_{H}+I_{H}+R_{H}\right)-\mu_{2}\left(S_{F}+I_{F}\right) \\
& -\mu_{3}\left(S_{R}+E_{R}+I_{R}\right)-\delta_{1} I_{H}-\delta_{2} I_{F}-\delta_{3} I_{R}-\omega_{1} A S_{H}-\omega_{2} A S_{R}-\mu_{4} A=0
\end{aligned}
$$

Substituting $N_{1}=S_{H}+E_{H}+I_{H}+R_{H}, \quad N_{2}=S_{F}+I_{F}$ and $N_{3}=S_{R}+E_{R}+I_{R}$ in (17) we have

$$
\psi_{1}+\psi_{2 s}+\psi_{2 i}+\psi_{3}-\mu_{1} N_{1}-\mu_{2} N_{2}-\mu_{3} N_{3}-\delta_{1} I_{H}-\delta_{2} I_{F}-\delta_{3} I_{R}+\lambda_{4}-\omega_{1} A S_{H}-\omega_{2} A S_{R}-\mu_{4} A=0
$$

But from Equation (16), we have $\lambda_{4}-\omega_{1} A S_{H}-\omega_{2} A S_{R}-\mu_{4} A=0$

It follows that

$$
\mu_{1} N_{1}+\mu_{2} N_{2}+\mu_{3} N_{3}+\delta_{1} I_{H}+\delta_{2} I_{F}+\delta_{3} I_{R}=\psi_{1}+\psi_{2 s}+\psi_{2 i}+\psi_{3}
$$

Since $\psi_{1}+\psi_{2 s}+\psi_{2 i}+\psi_{3}>0, \mu_{1}>0, \mu_{2}>0, \mu_{3}>0, \delta_{1}>0, \delta_{2}>0$ and $\delta_{3}>0$ we can discern that $\mu_{1} N_{1}>0, \mu_{2} N_{2}>0, \mu_{3} N_{3}>0, \delta_{1} I_{H}>0, \delta_{2} I_{F}>0$ and $\delta_{3} I_{R}>0$ implying that $S_{H}>0, E_{H}>0, I_{H}>0$, $S_{F}>0, I_{F}>0, S_{R}>0, E_{R}>0$ and $I_{R}>0$.

Hence endemic equilibrium point of the bubonic plague disease model in human, rodent, flea and pathogens in the environment exists.

Since the endemic equilibrium points exist, we now determine the conditions under which they are stable or unstable. We prove whether the solution starting sufficiently close to the equilibrium remains close to the equilibrium and approaches the equilibrium as $t \rightarrow \infty$, or if there are solutions starting arbitrary close to the equilibrium which do not approach it respectively.

\subsection{Global Stability of Endemic Equilibrium Point}

Using the idea from the study by [12] we say that the local stability of the Disease Free Equilibrium advocates for local stability of the Endemic Equilibrium for the reverse condition. We then work to find the global stability of Endemic equilibrium using a Korobeinikov approach as stipulated in [12]-[14] by forming a suitable Lyapunov function for our general model as given below:

We construct the Lyapunov function as given in the form:

$$
V=\sum a_{i}\left(y_{i}-y_{i}^{*} \ln y_{i}\right)
$$

where $a_{i}$ is defined as a properly selected positive constant, $y_{i}$ defines the population of the $i^{\text {th }}$ compartment, and $y_{i}^{*}$ is the equilibrium point.

We will have the following Lyapunov function,

$$
\begin{aligned}
V= & W_{1}\left(S_{H}-S_{H}^{*} \ln S_{H}\right)+W_{2}\left(E_{H}-E_{H}^{*} \ln E_{H}\right)+W_{3}\left(I_{H}-I_{H}^{*} \ln I_{H}\right) \\
& +W_{4}\left(R_{H}-R_{H}^{*} \ln R_{H}\right)+W_{5}\left(S_{R}-S_{R}^{*} \ln S_{R}\right)+W_{6}\left(E_{R}-E_{R}^{*} \ln E_{R}\right) \\
& +W_{7}\left(I_{R}-I_{R}^{*} \ln I_{R}\right)+W_{8}\left(S_{F}-S_{F}^{*} \ln S_{F}\right)+W_{9}\left(I_{F}-I_{F}^{*} \ln I_{F}\right) \\
& +W_{10}\left(A-A^{*} \ln A\right)
\end{aligned}
$$


The constants $W_{i}$ are non negative in $\Phi$ such that $W_{i}>0$ for $i=1,2,3, \cdots, 10$. The Lyapunov function $V$ together with its constants $W_{1}, W_{2}, \cdots, W_{10}$ chosen in such way that $V$ is continuous and differentiable in a space We then compute the time derivative of $V$ from it we get;

$$
\begin{aligned}
\frac{\mathrm{d} V}{\mathrm{~d} t}= & W_{1}\left(1-\frac{S_{H}^{*}}{S_{H}}\right) \frac{\mathrm{d} S_{H}}{\mathrm{~d} t}+W_{2}\left(1-\frac{E_{H}^{*}}{E_{H}}\right) \frac{\mathrm{d} E_{H}}{\mathrm{~d} t}+W_{3}\left(1-\frac{I_{H}^{*}}{I_{H}}\right) \frac{\mathrm{d} I_{H}}{\mathrm{~d} t}+W_{4}\left(1-\frac{R_{H}^{*}}{R_{H}}\right) \frac{\mathrm{d} R_{H}}{\mathrm{~d} t}+W_{5}\left(1-\frac{S_{R}^{*}}{S_{R}}\right) \frac{\mathrm{d} S_{R}}{\mathrm{~d} t} \\
& +W_{6}\left(1-\frac{E_{R}^{*}}{E_{R}}\right) \frac{\mathrm{d} E_{R}}{\mathrm{~d} t}+W_{7}\left(1-\frac{I_{R}^{*}}{I_{R}}\right) \frac{\mathrm{d} I_{R}}{\mathrm{~d} t}+W_{8}\left(1-\frac{S_{F}^{*}}{S_{F}}\right) \frac{\mathrm{d} S_{F}}{\mathrm{~d} t}+W_{9}\left(1-\frac{I_{F}^{*}}{I_{F}}\right) \frac{\mathrm{d} I_{F}}{\mathrm{~d} t}+W_{10}\left(1-\frac{A^{*}}{A}\right) \frac{\mathrm{d} A}{\mathrm{~d} t}
\end{aligned}
$$

Now using the general system (3)-(6) we will have

$$
\begin{aligned}
\frac{\mathrm{d} V}{\mathrm{~d} t}= & W_{1}\left(1-\frac{S_{H}^{*}}{S_{H}}\right)\left[\pi_{1} \psi_{1}+\varpi R_{H}-\alpha_{1}\left(\Gamma_{f h} \frac{I_{F}}{N_{2}}+\omega_{1} A\right) S_{H}-\mu_{1} S_{H}\right] \\
& +W_{2}\left(1-\frac{E_{H}^{*}}{E_{H}}\right)\left[\pi_{2} \psi_{1}+\alpha_{1}\left(\Gamma_{f h} \frac{I_{F}}{N_{2}}+\omega_{1} A\right) S_{H}-\alpha_{2} E_{H}-\mu_{1} E_{H}\right] \\
& +W_{3}\left(1-\frac{I_{H}^{*}}{I_{H}}\right)\left[\alpha_{2} E_{H}-\alpha_{3} I_{H}-\left(\mu_{1}+\delta_{1}\right) I_{H}\right] \\
& +W_{4}\left(1-\frac{R_{H}^{*}}{R_{H}}\right)\left[\pi_{3} \psi_{1}+\alpha_{3} I_{H}-\varpi R_{H}-\mu_{1} R_{H}\right] \\
& +W_{5}\left(1-\frac{S_{R}^{*}}{S_{R}}\right)\left[\kappa_{1} \psi_{3}-\gamma_{1}\left(\Gamma_{f r} \frac{I_{F}}{N_{2}}+\omega_{2} A\right) S_{R}-\mu_{3} S_{R}\right] \\
& +W_{6}\left(1-\frac{E_{R}^{*}}{E_{R}}\right)\left[\kappa_{2} \psi_{3}+\gamma_{1}\left(\Gamma_{f r} \frac{I_{F}}{N_{2}}+\omega_{2} A\right) S_{R}-\gamma_{2} E_{R}-\mu_{3} E_{R}\right] \\
& +W_{7}\left(1-\frac{I_{R}^{*}}{I_{R}}\right)\left[\kappa_{3} \psi_{3}+\gamma_{2} E_{R}-\left(\mu_{3}+\delta_{3}\right) I_{R}\right] \\
& +W_{8}\left(1-\frac{S_{F}^{*}}{S_{F}}\right)\left[\psi_{2 s}-\beta\left(\rho \Gamma_{h f} \frac{I_{H}}{N_{1}}+(1-\rho) \Gamma_{r f} \frac{I_{R}}{N_{3}}\right) S_{F}-\mu_{2} S_{F}\right] \\
& +W_{9}\left(1-\frac{I_{F}^{*}}{I_{F}}\right)\left[\psi_{2 i}+\beta\left(\rho \Gamma_{h f} \frac{I_{H}}{N_{1}}+(1-\rho) \Gamma_{r f} \frac{I_{R}}{N_{3}}\right) S_{F}-\left(\mu_{2}+\delta_{2}\right) I_{F}\right] \\
& +W_{10}\left(1-\frac{A^{*}}{A}\right)\left[\lambda_{4}-\omega_{1} A S_{H}-\omega_{2} A S_{R}-\mu_{4} A\right]
\end{aligned}
$$

At endemic equilibrium point we have

Human

$$
\begin{aligned}
& \pi_{1} \psi_{1}=-\varpi R_{H}^{*}+\alpha_{1}\left(\Gamma_{f h} \frac{I_{F}^{*}}{N_{2}^{*}}-\omega_{1} A^{*}\right) S_{H}^{*}+\mu_{1} S_{H}^{*}, \\
& \pi_{2} \psi_{1}=-\alpha_{1}\left(\Gamma_{f h} \frac{I_{F}^{*}}{N_{2}^{*}}-\omega_{1} A^{*}\right) S_{H}^{*}+\alpha_{2} E_{H}^{*}+\mu_{1} E_{H}^{*}, \\
& \alpha_{2}=\frac{1}{E_{H}^{*}}\left(\alpha_{3} I_{H}^{*}+\left(\mu_{1}+\delta_{1}\right) I_{H}^{*}\right) \\
& \pi_{3} \psi_{1}=-\alpha_{3} I_{H}^{*}+\varpi R_{H}^{*}+\mu_{1} R_{H}^{*}
\end{aligned}
$$

Rodent 


$$
\begin{aligned}
& \kappa_{1} \psi_{3}=\gamma_{1}\left(\Gamma_{f r} \frac{I_{F}^{*}}{N_{2}^{*}}-\omega_{2} A^{*}\right) S_{R}^{*}+\mu_{3} S_{R}^{*} \\
& \kappa_{2} \psi_{3}=-\gamma_{1}\left(\Gamma_{f r} \frac{I_{F}^{*}}{N_{2}^{*}}-\omega_{2} A^{*}\right) S_{R}^{*}+\gamma_{2} E_{R}^{*}+\mu_{3} E_{R}^{*} \\
& \kappa_{3} \psi_{3}=-\gamma_{2} E_{R}^{*}+\left(\mu_{3}+\delta_{3}\right) I_{R}^{*}
\end{aligned}
$$

Flea

$$
\begin{aligned}
& \psi_{2 s}=\beta\left(\rho \Gamma_{h f} \frac{I_{H}^{*}}{N_{1}^{*}}-(1-\rho) \Gamma_{r f} \frac{I_{R}^{*}}{N_{3}^{*}}\right) S_{F}^{*}+\mu_{2} S_{F}^{*} \\
& \psi_{2 i}=-\beta\left(\rho \Gamma_{h f} \frac{I_{H}^{*}}{N_{1}^{*}}-(1-\rho) \Gamma_{r f} \frac{I_{R}^{*}}{N_{3}^{*}}\right) S_{F}^{*}+\left(\mu_{2}+\delta_{2}\right) I_{F}^{*}
\end{aligned}
$$

where $\psi_{2 s}<\psi_{2 i}$

Pathogens

$$
\lambda_{4}=\omega_{1} A^{*} S_{H}^{*}+\omega_{2} A^{*} S_{R}^{*}+\mu_{4} A^{*}
$$

We can then rewrite $\frac{\mathrm{d} V}{\mathrm{~d} t}$ using (19), (20), (21) and (22) as:

$$
\begin{aligned}
& \frac{\mathrm{d} V}{\mathrm{~d} t}=W_{1}\left(1-\frac{S_{H}^{*}}{S_{H}}\right)\left[-\varpi R_{H}^{*}+\alpha_{1}\left(\Gamma_{f h} \frac{I_{F}^{*}}{N_{2}^{*}}-\omega_{1} A^{*}\right) S_{H}^{*}+\mu_{1} S_{H}^{*}+\varpi R_{H}-\alpha_{1}\left(\Gamma_{f h} \frac{I_{F}}{N_{2}}+\omega_{1} A\right) S_{H}-\mu_{1} S_{H}\right] \\
& +W_{2}\left(1-\frac{E_{H}^{*}}{E_{H}}\right)\left[-\alpha_{1}\left(\Gamma_{f h} \frac{I_{F}^{*}}{N_{2}^{*}}-\omega_{1} A^{*}\right) S_{H}^{*}+\alpha_{2} E_{H}^{*}+\mu_{1} E_{H}^{*}+\alpha_{1}\left(\Gamma_{f h} \frac{I_{F}}{N_{2}}+\omega_{1} A\right) S_{H}-\alpha_{2} E_{H}-\mu_{1} E_{H}\right] \\
& +W_{3}\left(1-\frac{I_{H}^{*}}{I_{H}}\right)\left[\left(\frac{1}{E_{H}^{*}}\left(\alpha_{3} I_{H}^{*}+\left(\mu_{1}+\delta_{1}\right) I_{H}^{*}\right)\right) E_{H}-\alpha_{3} I_{H}-\left(\mu_{1}+\delta_{1}\right) I_{H}\right] \\
& +W_{4}\left(1-\frac{R_{H}^{*}}{R_{H}}\right)\left[-\alpha_{3} I_{H}^{*}+\varpi R_{H}^{*}+\mu_{1} R_{H}^{*}+\alpha_{3} I_{H}-\varpi R_{H}-\mu_{1} R_{H}\right] \\
& +W_{5}\left(1-\frac{S_{R}^{*}}{S_{R}}\right)\left[\gamma_{1}\left(\Gamma_{\text {fr }} \frac{I_{F}^{*}}{N_{2}^{*}}-\omega_{2} A^{*}\right) S_{R}^{*}+\mu_{3} S_{R}^{*}-\gamma_{1}\left(\Gamma_{\text {fr }} \frac{I_{F}}{N_{2}}+\omega_{2} A\right) S_{R}-\mu_{3} S_{R}\right] \\
& +W_{6}\left(1-\frac{E_{R}^{*}}{E_{R}}\right)\left[-\gamma_{1}\left(\Gamma_{f r} \frac{I_{F}^{*}}{N_{2}^{*}}-\omega_{2} A^{*}\right) S_{R}^{*}+\gamma_{2} E_{R}^{*}+\mu_{3} E_{R}^{*}+\gamma_{1}\left(\Gamma_{f r} \frac{I_{F}}{N_{2}}+\omega_{2} A\right) S_{R}-\gamma_{2} E_{R}-\mu_{3} E_{R}\right] \\
& +W_{7}\left(1-\frac{I_{R}^{*}}{I_{R}}\right)\left[-\gamma_{2} E_{R}^{*}+\left(\mu_{3}+\delta_{3}\right) I_{R}^{*}+\gamma_{2} E_{R}-\left(\mu_{3}+\delta_{3}\right) I_{R}\right] \\
& +W_{8}\left(1-\frac{S_{F}^{*}}{S_{F}}\right)\left[\beta\left(\rho \Gamma_{h f} \frac{I_{H}^{*}}{N_{1}^{*}}-(1-\rho) \Gamma_{r f} \frac{I_{R}^{*}}{N_{3}^{*}}\right) S_{F}^{*}+\mu_{2} S_{F}^{*}-\beta\left(\rho \Gamma_{h f} \frac{I_{H}}{N_{1}}+(1-\rho) \Gamma_{r f} \frac{I_{R}}{N_{3}}\right) S_{F}-\mu_{2} S_{F}\right] \\
& +W_{9}\left(1-\frac{I_{F}^{*}}{I_{F}}\right)\left[-\beta\left(\rho \Gamma_{h f} \frac{I_{H}^{*}}{N_{1}^{*}}-(1-\rho) \Gamma_{r f} \frac{I_{R}^{*}}{N_{3}^{*}}\right) S_{F}+\left(\mu_{2}+\delta_{2}\right) I_{F}^{*}\right. \\
& \left.+\beta\left(\rho \Gamma_{h f} \frac{I_{H}}{N_{1}}+(1-\rho) \Gamma_{r f} \frac{I_{R}}{N_{3}}\right) S_{F}-\left(\mu_{2}+\delta_{2}\right) I_{F}\right] \\
& +W_{10}\left(1-\frac{A^{*}}{A}\right)\left[\omega_{1} A^{*} S_{H}^{*}+\omega_{2} A^{*} S_{R}^{*}+\mu_{4} A^{*}-\omega_{1} A S_{H}-\omega_{2} A S_{R}-\mu_{4} A\right]
\end{aligned}
$$


After simplification the above equation becomes:

$$
\begin{aligned}
\frac{\mathrm{d} V}{\mathrm{~d} t}= & -W_{1}\left(1-\frac{S_{H}^{*}}{S_{H}}\right)^{2}-W_{2}\left(1-\frac{E_{H}^{*}}{E_{H}}\right)^{2}-W_{3}\left(1-\frac{I_{H}^{*}}{I_{H}}\right)^{2} \\
& -W_{4}\left(1-\frac{R_{H}^{*}}{R_{H}}\right)^{2}-W_{5}\left(1-\frac{S_{R}^{*}}{S_{R}}\right)^{2}-W_{6}\left(1-\frac{E_{R}^{*}}{E_{R}}\right)^{2} \\
& -W_{7}\left(1-\frac{I_{R}^{*}}{I_{R}}\right)^{2}-W_{8}\left(1-\frac{S_{F}^{*}}{S_{F}}\right)^{2}-W_{9}\left(1-\frac{I_{F}^{*}}{I_{F}}\right)^{2} \\
& -W_{10}\left(1-\frac{A^{*}}{A}\right)^{2}+F\left(S_{H}, E_{H}, I_{H}, R_{H}, S_{R}, E_{R}, I_{R}, S_{F}, I_{F}, A\right)
\end{aligned}
$$

where the function $F\left(S_{H}, E_{H}, I_{H}, R_{H}, S_{R}, E_{R}, I_{R}, S_{F}, I_{F}, A\right)$ is non positive, Now following the procedures by [15] [16]. We have $F\left(S_{H}, E_{H}, I_{H}, R_{H}, S_{R}, E_{R}, I_{R}, S_{F}, I_{F}, A\right) \leq 0$ for all $S_{H}, E_{H}, I_{H}, R_{H}, S_{R}, E_{R}, I_{R}, S_{F}, I_{F}, A$, Then $\frac{\mathrm{d} V}{\mathrm{~d} t} \leq 0$ for all $S_{H}, E_{H}, I_{H}, R_{H}, S_{R}, E_{R}, I_{R}, S_{F}, I_{F}, A$ and it is zero when $S_{H}=S_{H}^{*}, E_{H}=E_{H}^{*}, I_{H}=I_{H}^{*}$, $R_{H}=R_{H}^{*}, S_{R}=S_{R}^{*}, E_{R}=E_{R}^{*}, I_{R}=I_{R}^{*}, S_{F}=S_{F}^{*}, I_{F}=I_{F}^{*}, A=A^{*}$ Hence the largest compact invariant set in $S_{H}, E_{H}, I_{H}, R_{H}, S_{R}, E_{R}, I_{R}, S_{F}, I_{F}, A$ such that $\frac{\mathrm{d} V}{\mathrm{~d} t}=0$ is the singleton $E^{*}$ which is Endemic Equilibrium point of the model system (3)-(6).

LaSalles's invariant principle by [17] then implies that $E^{*}$ is globally asymptotically stable in the interior of the region of $S_{H}, E_{H}, I_{H}, R_{H}, S_{R}, E_{R}, I_{R}, S_{F}, I_{F}, A$ and thus leads to the Theorem 3.

Theorem 3. If $R_{0}>1$ then the bubonic plague disease model system (3)-(6) has a unique endemic equilibrium point $E^{*}$ which is globally asymptotically stable in $S_{H}, E_{H}, I_{H}, R_{H}, S_{R}, E_{R}, I_{R}, S_{F}, I_{F}, A$.

\section{Numerical Simulation}

Numerical simulation is carried out in order to observe and understand the kinetics of bubonic plague disease and demonstrate analytical results. In particular we illustrate through numerical simulation the stability of the endemic equilibrium states in human, rodent, flea and pathogens in the environment.

\section{Parameter Values}

The values of the parameters used in bubonic plague disease model are shown in Table 2. The parameters are taken from the previous studies that relate to this study, existing information and through estimation.

In the simulation we assume different cases where each sub-population starts at different initial values (six different initial values) ultimately returns to its endemic point. We thus justify that a solution that starts sufficiently close to the equilibrium remains close to it and it eventually approaches the equilibrium as $t \rightarrow \infty$.

Figure 1 shows the dynamical behavior of the human population. The sub-Figure 1(a) shows a marginal increase in number of susceptible human as people moves in through migration. When the disease becomes endemic, the number of susceptible human decreases as they becomes exposed to the disease due to the increase of force of infection which resembles to the general scenario of vector borne infection as depicted in [23]. Given that the model assumes no treatment nor vaccination is applied, it thus justifies the behavior illustrated in sub-Figure 1(b). The figure shows the very slight increase of a exposed human beings before it drops to its endemic level as the large number of exposed human progresses and become infected human. The increase of number of infected human beings from the exposed class is depicted in sub-Figure $\mathbf{1}(\mathrm{c})$. We can see that in the first five years the number infected human subgroup experience a substantial increase before it decreases to its endemic level. The decrease in number of infected human is mainly through natural death and disease induced death whereas very few will recover and join a recovery class. The system considers only natural recovery (recovery due to individual's strong body immunity), thus the number of recovery human will slightly increase before it decreases and reaches its endemic level as illustrated in sub-Figure 1(d) [24] [25]. 
Table 2. Parameters values for Bubonic Plague disease model.

\begin{tabular}{|c|c|c|}
\hline Parameters & Value/Range & Reference/Source \\
\hline$\Gamma_{r f}$ & 0.6 & Estimated \\
\hline$\Gamma_{f h}$ & 0.09 & [18] \\
\hline$\Gamma_{f r}$ & 4.7 & [19] \\
\hline$\alpha_{1}$ & 0.9 & Estimated \\
\hline$\gamma_{1}$ & 0.9 & Estimated \\
\hline$\Gamma_{h f}$ & 0.28 & [18] \\
\hline$\lambda_{4}$ & 0.89 & Estimated \\
\hline$\alpha_{2}$ & 0.04 & [20] \\
\hline$\gamma_{2}$ & 0.05 & [20] \\
\hline$\alpha_{3}$ & 0.1 & [20] \\
\hline$\varpi$ & 0.1 & {$[20]$} \\
\hline$\mu_{1}$ & 0.04 & [20] \\
\hline$\delta_{1}$ & 0.04 & [20] \\
\hline$\delta_{3}$ & 0.05 & [21] \\
\hline$\mu_{3}$ & 0.2 & {$[22]$} \\
\hline$\omega_{1}$ & 0.01 & {$[20]$} \\
\hline$\omega_{2}$ & 0.073 & [18] \\
\hline$\mu_{4}$ & 0.1 & Estimated \\
\hline$\mu_{2}$ & 0.07 & [18] \\
\hline$\delta_{2}$ & 0.03 & [18] \\
\hline$\psi_{1}$ & 0.09 & Estimated \\
\hline$\psi_{2 s}$ & 0.008 & [21] \\
\hline$\psi_{2 i}$ & 0.08 & Estimated \\
\hline$\psi_{3}$ & 0.03 & [20] \\
\hline$\beta$ & 0.99 & Estimated \\
\hline
\end{tabular}

Figure 2 shows the dynamics in rodent population. The results seen in this figure also settles with the findings by [26] [27]. We can see from sub-Figure 2(a) that the susceptible rodent population drops very fast within the first year, before it slightly rise due to migration at the rate $\kappa_{1} \psi_{3}$, to its endemic equilibrium level. The quick drop of susceptible rodent may be due to the fact that rodents are the primary victim of bubonic plague so that when the disease is endemic most of them are infected and become exposed to the disease [28]. The increase of the rate of infection in susceptible rodent population proportionally increase the number of exposed rodent [29]. After the significant increase of the exposed rodent population within the first five years it then drops to its endemic level. It takes only 2 to 6 days for an exposed rodent to become infectious [30] which is the reason for a quick decrease of exposed rodent as seen in sub-Figure 2(b). The infectious rodent population increases as the number of rodent progressing from exposed class to infectious increase. $I_{R}$ then drops to its endemic level as it experience both natural and disease induced death as in sub-Figure 2(c). 


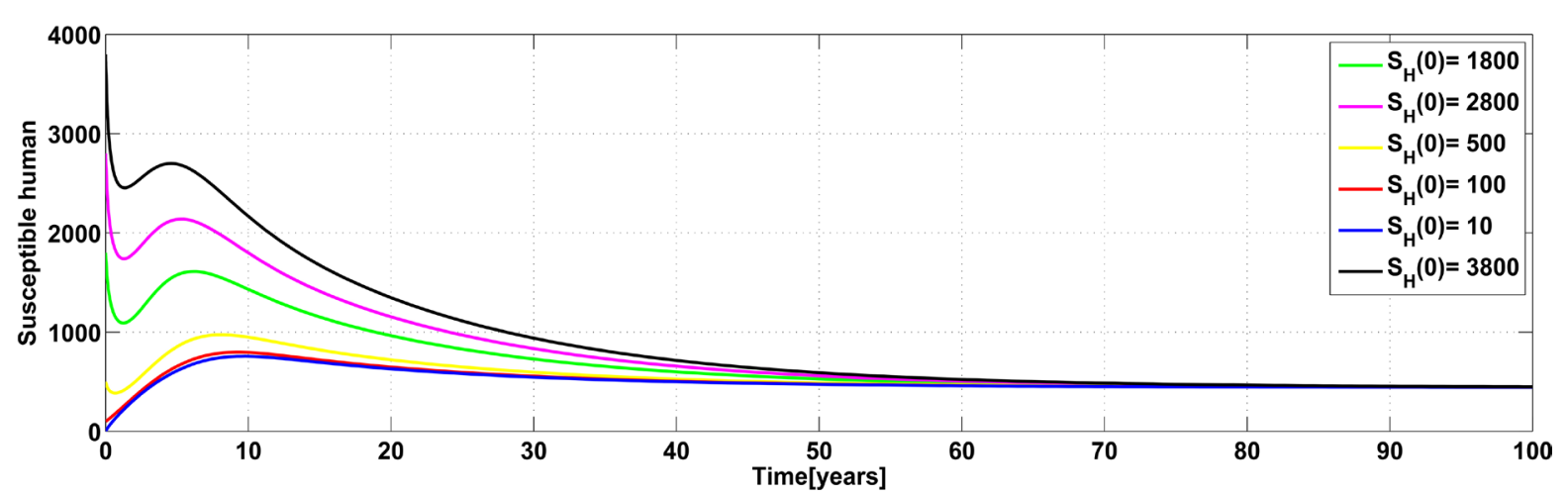

(a)

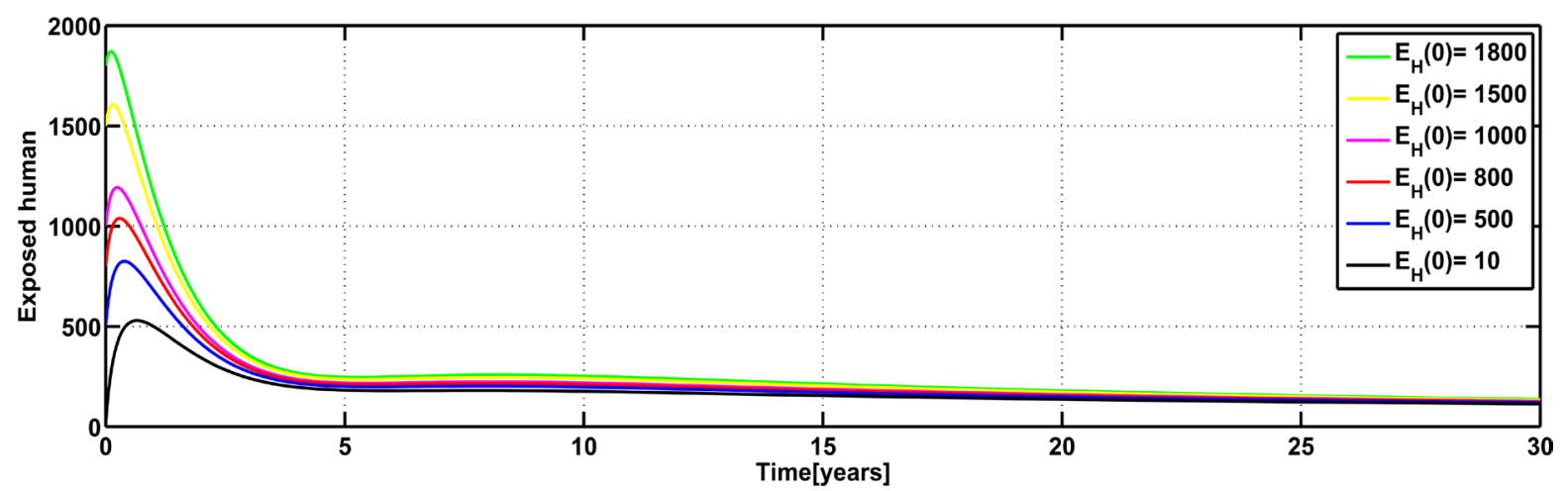

(b)

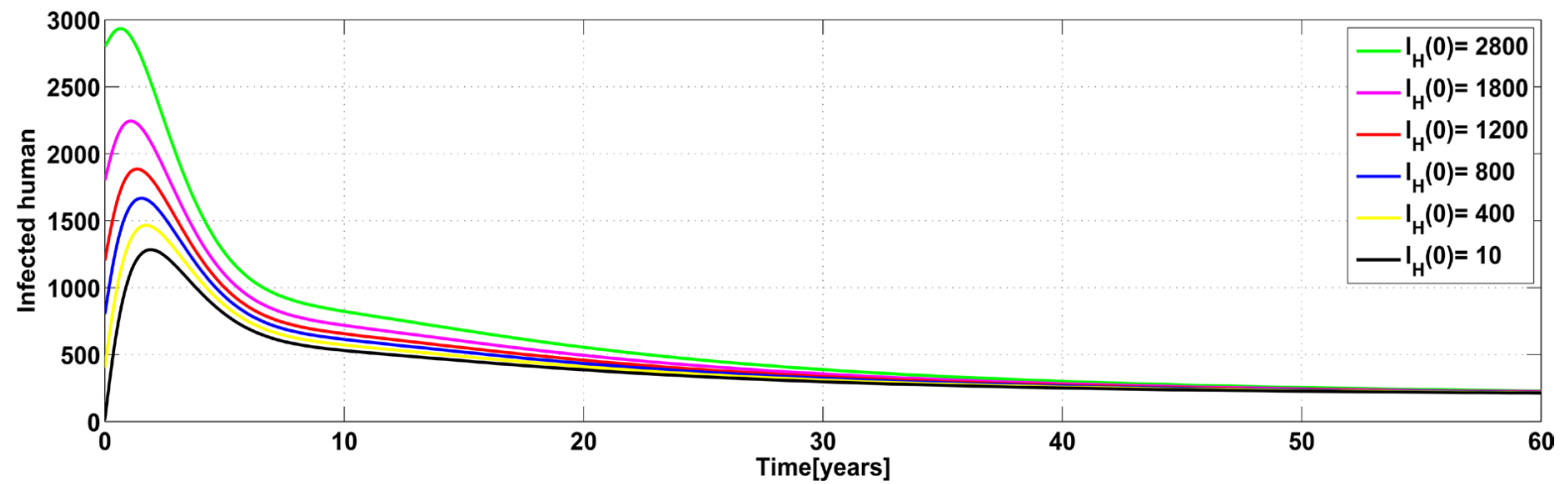

(c)

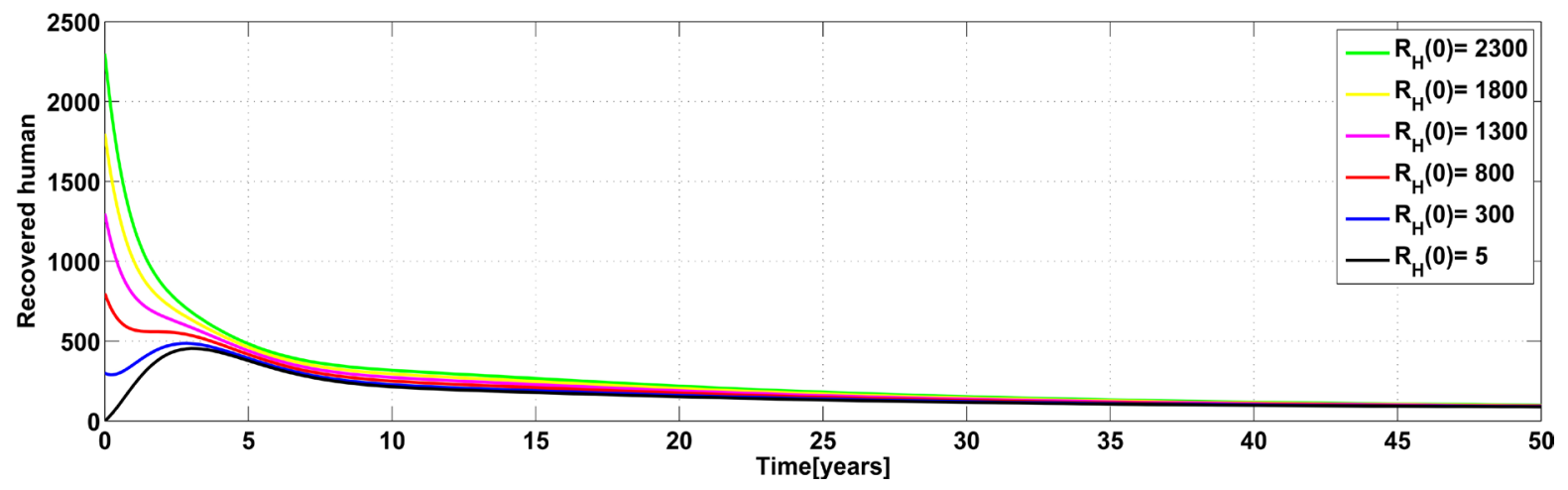

(d)

Figure 1. Simulation of the model's solution trajectories to show stability of the endemic point in subsystem (3). 


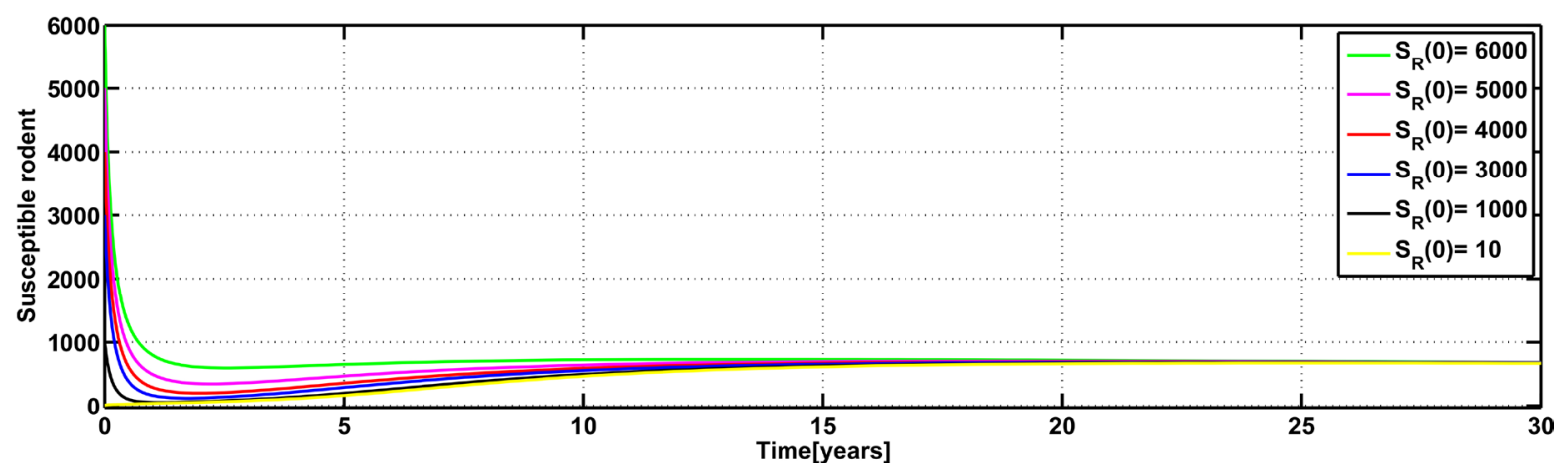

(a)

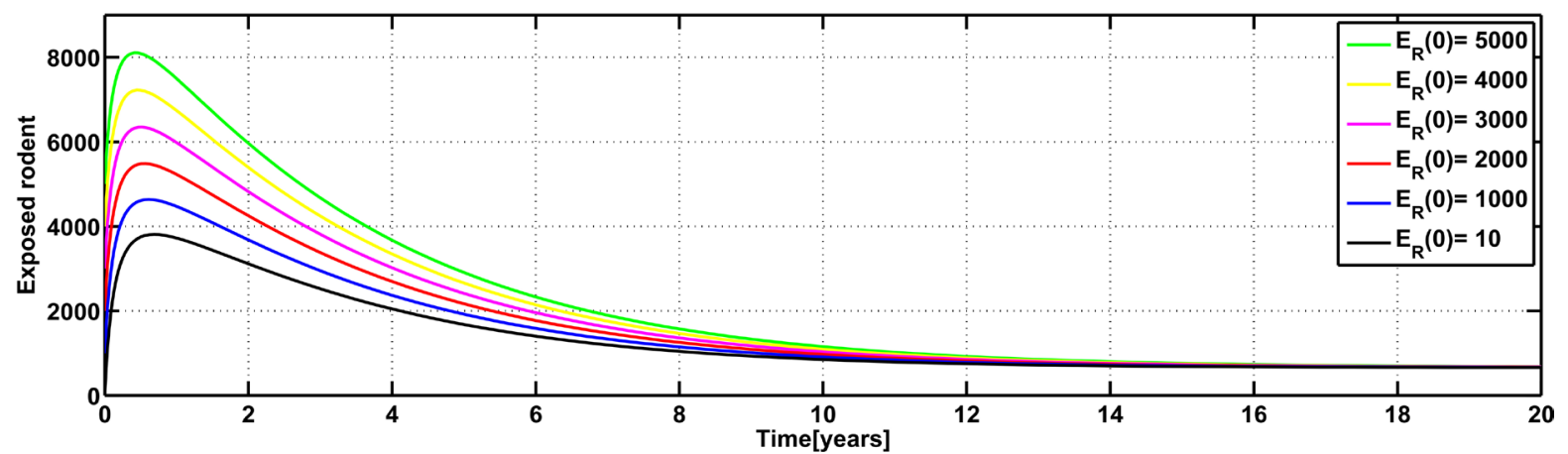

(b)

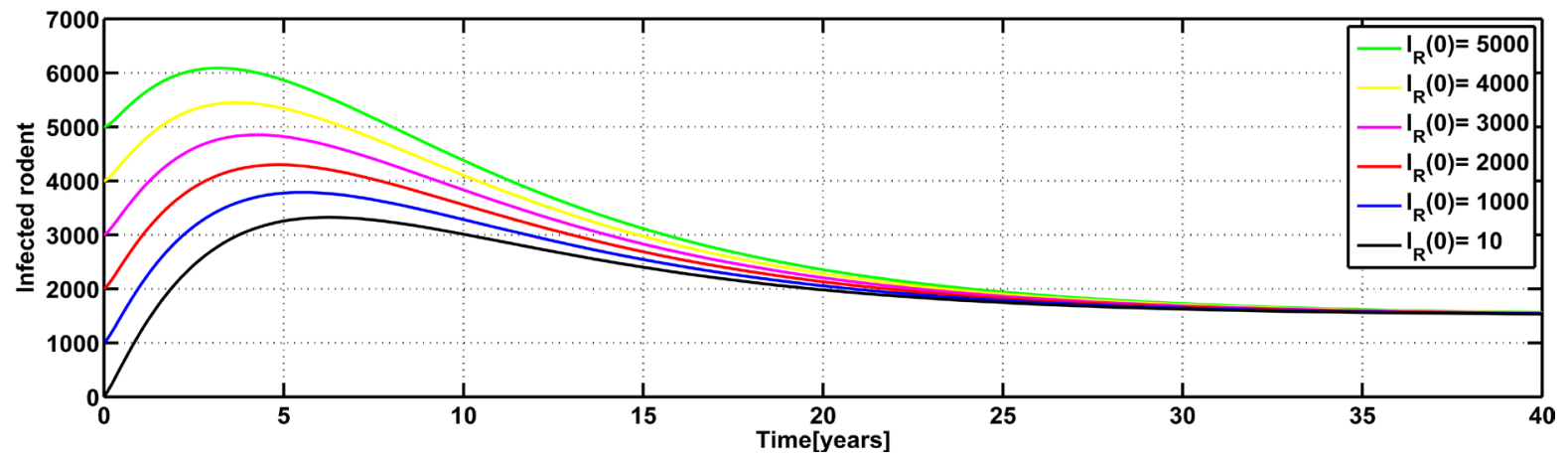

(c)

Figure 2. Simulation of the model's solution trajectories to show stability of the endemic point in subsystem (4).

The dynamics in the flea population are as seen in Figure 3, we can see that the number of susceptible flea decreases exponentially as they die naturally or acquire infection from the infected rodent or human at the rate $\Gamma_{h f}$ or $\Gamma_{r f}$ respectively see sub-Figure 3(a). The increased death of rodent due to the endemicity of the disease, will as a result lead to scarcity of hosts for flea to feed on and thus die [26]. The addition of natural and disease induced death in infected flea population will lead to a quick drops to its endemic level as illustrated in sub-Figure 3(b) (this corresponds well with the findings in the study by [20] [31]). The pathogens in the environment are removed when they come to contact with the susceptible human and rodent at the rate $\omega_{1}$ and $\omega_{2}$ respectively and due to natural death at the rate $\mu_{4}$. Since we assume that human and rodent infectious classes have a negligible contribution in increasing the number of pathogens in the environment (see Equation (6)). Now as the disease become endemic the rates $\omega_{1}$ and $\omega_{2}$ increase which in turn decrease the number of pathogens in the environment. Pathogens are also highly affected by the condition in the environment (temperature, humidity and precipitation). Most of the time this lead to a massive decay of the pathogens population in the environment as the environment is not favorable for their survival and growth [32] Then the number of pathogens in the environment will gradually decrease to its endemic level as in Figure 4. 


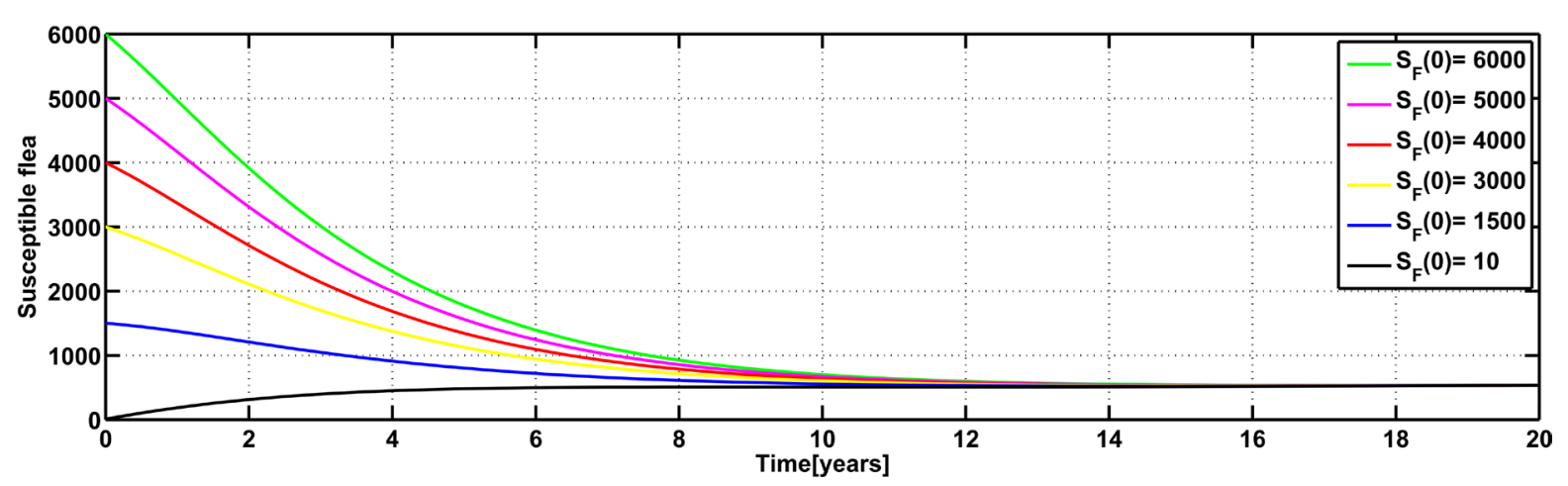

(a)

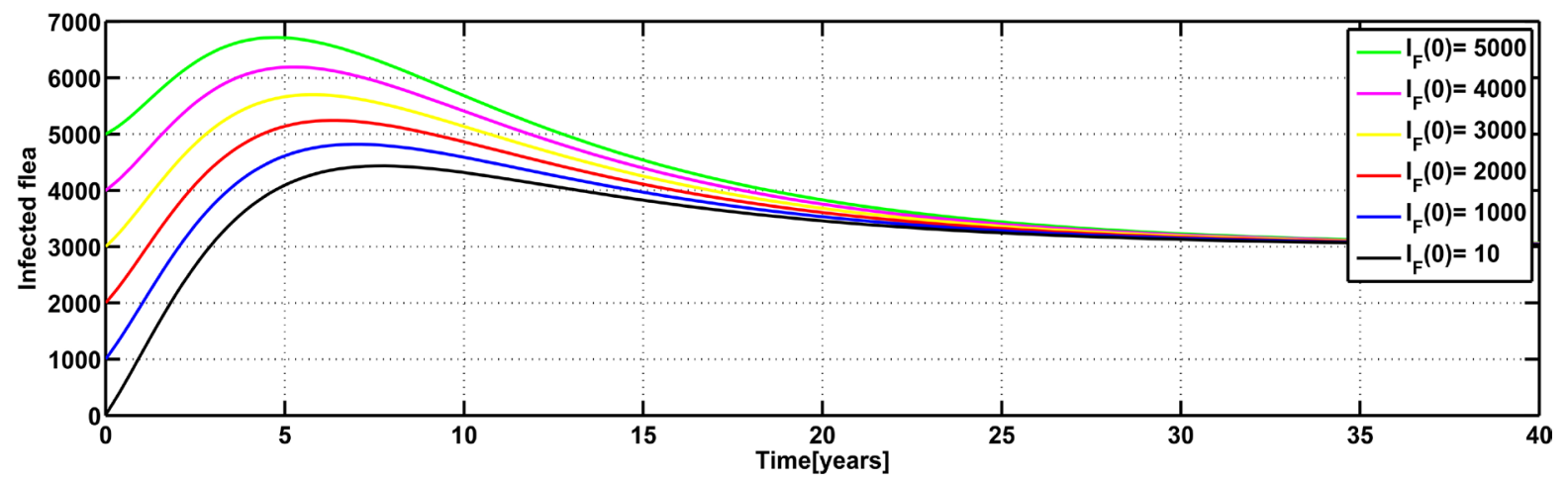

(b)

Figure 3. Simulation of the model's solution trajectories to show stability of the endemic point of subsystem (5).

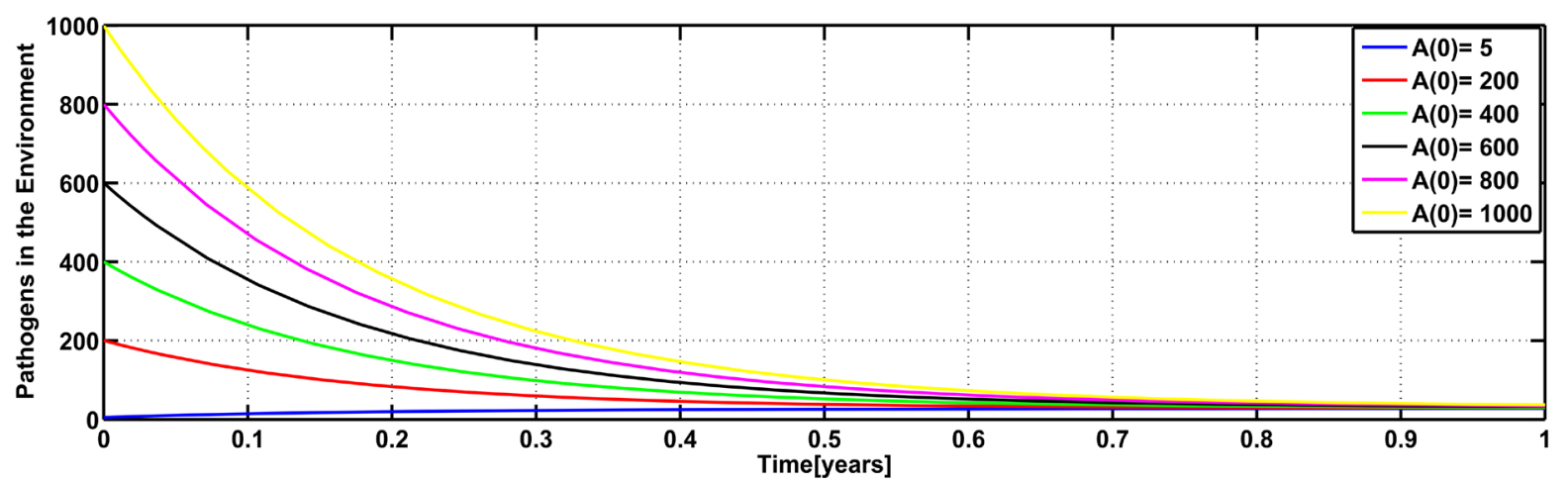

Figure 4. Simulation of the model's solution trajectories to show stability of the endemic point in (6).

\section{Conclusion}

In this paper, we have considered a bubonic plague in human, rodent and flea with Yersinia pestis in the environment. We have carried out the stability analysis of the equilibrium states in which the analytical results show that the disease free equilibrium point is locally and globally asymptotically stable when $R_{0}<0$ and unstable when $R_{0}>0$. This result necessitates that the basic reproduction number, which is the expected number of secondary cases produced by a single infected individual during the entire infectious period of that particular individual in a completely susceptible population is a key non-dimension parameter that dictates whether the disease will spread or die out. When $R_{0}$ is increased or decreased above or below unity compels to the persistence or eradication of bubonic plague disease respectively. The decrease or increase of the basic reproduction number will as a result affects negatively or positively the flea's infective period $\frac{1}{\mu_{2}+\delta_{2}}$, probability that rodent sur- 
vive the infected class $\frac{\gamma_{2}}{\mu_{3}+\gamma_{2}}$, the adequate contact rate flea to human $\Gamma_{f h}$, rodent's infective period $\frac{1}{\mu_{3}+\delta_{3}}$, the probability that flea gets the disease from the rodent or human which are $(1-\rho) \Gamma_{r f}$ or $\rho \Gamma_{h f}$ respectively. The human infective period $\frac{1}{\mu_{1}+\delta_{1}+\alpha_{3}}$, probability that human survive the infected class $\frac{\alpha_{2}}{\mu_{1}+\alpha_{2}}$, the rate at which fleas gets infected $\beta$, the adequate contact rate flea to rodent $\Gamma_{f r}$ and the rate at which human and rodent become exposed to the the disease which are $\alpha_{1}$ and $\gamma_{1}$ respectively. The endemic equilibrium point is also found to be locally and globally asymptotically stable whenever they exist. Using the model's parameters values from literature reviewed in this paper and some estimated, we use the simulation to show the endemic equilibrium for Human, Rodent, Flea and pathogens in the environment are stable thus supports the analytical results. We observe that without intervention that controls the value of $R_{0}$ to less than a unity bubonic plague may be very fatal and a life threatening disease whenever it occurs.

\section{Acknowledgements}

We thank the Editor and the referee for their comments. Research of R. C. Ngeleja is funded by The Government of Tanzania through Nelson Mandela African Institution of Science and Technology (NM-AIST). This support is greatly appreciated.

\section{References}

[1] Gonzalez, R.J. and Miller, V.L. (2016) A Deadly Path: Bacterial Spread during Bubonic Plague. Trends in Microbiology, 24, 239-241. http://dx.doi.org/10.1016/j.tim.2016.01.010

[2] Guinet, F., Avffe, P., Filali, S., Huon, C., Savin, C., Huerre, M., Fiette, L. and Carniel, E. (2015) Dissociation of Tissue Destruction and Bacterial Expansion during Bubonic Plague. PLoS Pathogens, 11, e1005222. http://dx.doi.org/10.1371/journal.ppat.1005222

[3] Eisen, R.J., Dennis, D.T. and Gage, K.L. (2015) The Role of Early-Phase Transmission in the Spread of Yersinia pestis. Journal of Medical Entomology, 52, 1183-1192. http://dx.doi.org/10.1093/jme/tjv128

[4] Ayyadurai, S., Houhamdi, L., Lepidi, H., Nappez, C., Raoult, D. and Drancourt, M. (2008) Long-Term Persistence of Virulent Yersinia Pestis in Soil. Microbiology, 154, 2865-2871. http://dx.doi.org/10.1099/mic.0.2007/016154-0

[5] Eisen, R.J., Petersen, J.M., Higgins, C.L., Wong, D., Levy, C.E., Mead, P.S., Schriefer, M.E., Griffth, K.S., Gage, K.L. and Beard, C.B. (2008) Persistence of Yersinia pestis in Soil under Natural Conditions. Emerging Infectious Diseases, 14, 941. http://dx.doi.org/10.3201/eid1406.080029

[6] Ngeleja, R.C., Luboobi, L.S. and Nkansah-Gyekye, Y. (2016) Modelling the Dynamics of Bubonic Plague with Yersinia pestis in the Environment. Communications in Mathematical Biology and Neuroscience, 2016, 5-10.

[7] Martcheva, M. (2015) Introduction to Mathematical Epidemiology. Vol. 61, Springer. http://dx.doi.org/10.1007/978-1-4899-7612-3

[8] Li, J., Blakeley, D. and Smith, R. (2011) The Failure of r0. Computational and Mathematical Methods in Medicine, 2011. Article ID: 527610.

[9] Castillo-Chavez, C., Blower, S., Driessche, P., Kirschner, D. and Yakubu, A.-A. (2002) Mathematical Approaches for Emerging and Reemerging Infectious Diseases: Models, Methods, and Theory. Springer. http://dx.doi.org/10.1007/978-1-4757-3667-0

[10] Tumwiine, J., Mugisha, J. and Luboobi, L.S. (2007) A Mathematical Model for the Dynamics of Malaria in a Human Host and Mosquito Vector with Temporary Immunity. Applied Mathematics and Computation, 189, 1953-1965. http://dx.doi.org/10.1016/j.amc.2006.12.084

[11] Massawe, L.N., Massawe, E.S. and Makinde, O.D. (2015) Temporal Model for Dengue Disease with Treatment. Advances in Infectious Diseases, 5, 21. http://dx.doi.org/10.4236/aid.2015.51003

[12] Van den Driessche, P. and Watmough, J. (2002) Reproduction Numbers and Sub-Threshold Endemic Equilibria for Compartmental Models of Disease Transmission. Mathematical Biosciences, 180, 29-48.

http://dx.doi.org/10.1016/S0025-5564(02)00108-6

[13] Korobeinikov, A. (2004) Lyapunov Functions and Global Properties for Seir and Seis Epidemic Models. Mathematical 
Medicine and Biology, 21, 75-83. http://dx.doi.org/10.1093/imammb/21.2.75

[14] Korobeinikov, A. (2007) Global Properties of Infectious Disease Models with Nonlinear Incidence. Bulletin of Mathematical Biology, 69, 1871-1886. http://dx.doi.org/10.1007/s11538-007-9196-y

[15] McCluskey, C. (2006) Lyapunov Functions for Tuberculosis Models with Fast and Slow Progression. Mathematical Biosciences and Engineering: MBE, 3, 603-614. http://dx.doi.org/10.3934/mbe.2006.3.603

[16] Korobeinikov, A. and Wake, G.C. (2002) Lyapunov Functions and Global Stability for Sir, Sirs, and Sis Epidemiological Models. Applied Mathematics Letters, 15, 955-960. http://dx.doi.org/10.1016/S0893-9659(02)00069-1

[17] La Salle, J. (1976) The Stability of Dynamical Systems. SIAM.

[18] Benkirane, S., Shankland, C., Norman, R. and McCaig, C. (2009) Modelling the Bubonic Plague in a Prairie Dog Burrow: A Work in Progress. 8th Workshop on Process Algebra and Stochastically Timed Activities, Edinburgh, 26-27 August 2009, 145-152.

[19] Li, W.-H. (1993) Unbiased Estimation of the Rates of Synonymous and Nonsynonymous Substitution. Journal of Molecular Evolution, 36, 96-99. http://dx.doi.org/10.1007/BF02407308

[20] Keeling, M. and Gilligan, C. (2000) Bubonic Plague: A Metapopulation Model of a Zoonosis. Proceedings of the Royal Society of London Series B: Biological Sciences, 267, 2219-2230. http://dx.doi.org/10.1098/rspb.2000.1272

[21] Keeling, M. and Gilligan, C. (2000) Metapopulation Dynamics of Bubonic Plague. Nature, 407, 903-906. http://dx.doi.org/10.1038/35038073

[22] Galtier, N. and Mouchiroud, D. (1998) Isochore Evolution in Mammals: A Human-Like Ancestral Structure. Genetics, 150, 1577-1584.

[23] Lemon, S.M., Sparling, P.F., Hamburg, M.A., Relman, D.A., Choffnes, E.R., Mack, A., et al. (2008) Vectorborne Diseases: Understanding the Environmental, Human Health, and Ecological Connections, Workshop Summary (Forum on Microbial Threats). National Academies Press, Washington DC.

[24] McNeill, W. (2010) Plagues and Peoples. Anchor, Norwell.

[25] Poland, J.D. and Dennis, D.T. (1998) Plague. In: Evans, A.S. and Brachman, P.S., Eds., Bacterial Infections of Humans, Springer, Berlin, 545-558. http://dx.doi.org/10.1007/978-1-4615-5327-4_28

[26] Gage, K.L. and Kosoy, M.Y. (2005) Natural History of Plague: Perspectives from More than a Century of Research. Annual Review of Entomology, 50, 505-528. http://dx.doi.org/10.1146/annurev.ento.50.071803.130337

[27] Scott, S. and Duncan, C.J. (2001) Biology of Plagues: Evidence from Historical Populations. Cambridge University Press, Cambridge. http://dx.doi.org/10.1017/CBO9780511542527

[28] Dennis, D.T. and Staples, J.E. (2009) Plague. In: Evans, A.S. and Brachman, P.S., Eds., Bacterial Infections of Humans, Springer, Berlin, 597-611. http://dx.doi.org/10.1007/978-0-387-09843-2 28

[29] Davis, S. and Calvet, E. (2005) Fluctuating Rodent Populations and Risk to Humans from Rodent-Borne Zoonoses. Vector-Borne \& Zoonotic Diseases, 5, 305-314. http://dx.doi.org/10.1089/vbz.2005.5.305

[30] Putzker, M., Sauer, H. and Sobe, D. (2000) Plague and Other Human Infections Caused by Yersinia Species. Clinical Laboratory, 47, 453-466.

[31] Samia, N.I., Kausrud, K.L., Heesterbeek, H., Ageyev, V., Begon, M., Chan, K.-S. and Stenseth, N.C. (2011) Dynamics of the Plague-Wildlife-Human System in Central Asia Are Controlled by Two Epidemiological Thresholds. Proceedings of the National Academy of Sciences of the United States of America, 108, 14527-14532. http://dx.doi.org/10.1073/pnas.1015946108

[32] Ari, T.B., Neerinckx, S., Gage, K.L., Kreppel, K., Laudisoit, A., Leirs, H. and Stenseth, N.C. (2011) Plague and Climate: Scales Matter. PLoS Pathogens, 7, e1002160. http://dx.doi.org/10.1371/journal.ppat.1002160 


\section{Submit or recommend next manuscript to SCIRP and we will provide best service for you:}

Accepting pre-submission inquiries through Email, Facebook, LinkedIn, Twitter, etc.

A wide selection of journals (inclusive of 9 subjects, more than 200 journals)

Providing 24-hour high-quality service

User-friendly online submission system

Fair and swift peer-review system

Efficient typesetting and proofreading procedure

Display of the result of downloads and visits, as well as the number of cited articles

Maximum dissemination of your research work

Submit your manuscript at: http://papersubmission.scirp.org/ 